\title{
Probabilistic analysis of Improved Austin-Moore stem used in cementless total hip arthroplasty considering loading uncertainty
}

\author{
Analyse probabiliste de la tige améliorée Austin-Moore utilisée dans \\ l'arthroplastie totale de la hanche sans ciment compte tenu de
}

l'incertitude de chargement

\author{
Ghais Kharmanda ${ }^{1}$ \\ ${ }^{1}$ Mechanics Laboratory of Normandy, INSA Rouen, St Etienne du Rouvray, France
}

\begin{abstract}
Austin-Moore hemiarthroplasty had been critically utilized for aged patients with femoral neck fractures. However, this implant became no longer favorable when increasing life activity. A multiobjective shape optimization has been integrated to improve its performance. The resulting configuration is called Improved Austin-Moore (IAM) model. Probabilistic analysis is very important when the input data are random, that leads to stochastic results. In this paper, a probabilistic analysis is applied to solid and IAM stems implanted in a proximal femur in order to show their advantages. This way it is possible to control the biomechanical effects of the implanted femur to determine its performance. The applied loads are generated randomly using Monte Carlo Simulations (MCS). MSC sampling technique is applied and the different von-Mises stresses of the layers (bone and metal) are selected as performance indicators. Two simple 2D implant-bone models of the solid and IAM designs are studied with a target reliability index equals to $\beta_{t}=3$, which corresponds to a high level of confidence (reliability) $99.87 \%$. The major finding of this article is that the skewness values of all output parameters of the IAM stem are positive which means that the majority of the maximum von-Mises stress values are closer to their minimum values than those associated with the solid stem. In addition, the sensitivity analysis shows that the input parameters for the IAM stem are more effective on the output parameters relative to those associated with the solid stem. The IAM stem shows a high interdependence (correlation) between the input and output parameters when comparing with the solid stem. Since this study is carried out considering loading uncertainty, the geometry can affect the load transfer. Therefore, a correlation study between the input parameters is carried out and showed significant coefficient values for the IAM stem relative to the solid one. The results show that the IAM stem is much more advantageous than the solid stem.

KEYWORDS. Hip Prosthesis, Probabilistic Analysis, Finite Element Analysis, Monte Carlo Simulations, IAM stem.
\end{abstract}

\section{Introduction}

This work is a continuation of the previous work of the integration of multi-objective structural optimization into cementless hip prosthesis design published by Kharmanda (2016), in which a new stem design, so-called Improved Austin-Moore (IAM) is developed. In this work, a probabilistic analysis of the IAM stem used in cementless total hip replacement considering loading uncertainty is carried out in order to show the advantages of the IAM stem relative to the solid one.

The high complication rates following osteoporotic femoral neck fractures can leads to destructive effects on the patient and increase the burden on health care systems. The number of the total hip replacement number is increasing accordingly (Huang et al. 2016; Huang et al. 2019). Austin-Moore prostheses had been selectively utilized for the total hip arthroplasty. According to several studies, the failure rate of the Austin-Moore prosthesis has been found to be $2-10 \%$ at midterm follow-up and 6-35\% at long-term follow-up (Sadoghi et al. 2013; Kanto et al. 2014). There is a strong need to decrease complications related to this total hip arthroplasty. 
When performing the probabilistic studies on total hip replacement models, it has been shown that there are many important random variables, such as bone geometry, implant design geometry, cement geometry, material properties, magnitude and direction of applied loads, and bone-implant relative position that may affect performance (Gonzalez 2009; Kharmanda and Antypas 2018). Several considerations have been taken into account with aim of improving the stem design. First, to consider the rectangular section stem instead for the circular one, it can lead to much more reliable designs (Kharmanda et al. 2017). Gonzalez (2009) showed that the rectangular section stem allows a secure diaphyseal press-fit in the frontal plane of the femoral canal, which provides excellent rotational stability and increases primary mechanical fixation. In Kharmanda and Albashi (2011), it has been also shown that it can reduce the wear. Second, to consider the shouldered stem instead for the non-shouldered one, it can lead to a good reduction of the maximum von-Mises stress values in the different bone layers relative to the non-shouldered one (Kharmanda et al. 2012; Kharmanda et al. 2014). Third, it concerns the hollow stem instead for solid one, that leads to a good fixation and increases the prosthesis performance (Kharmanda and Ibrahim 2013; Kharmanda and El-Hami 2017; Kharmanda et al. 2019). In this context, Kharmanda (2016) integrated a multiobjective structural optimization strategy into Austin-Moore stem in order to improve its performance. The resulting configuration was called Improved Austin-Moore (IAM). The topology optimization was considered as a conceptual design aspect to sketch the IAM stem according to the daily loading cases. The shape optimization presented the detailed design aspect considering several objectives. The proposed multiplicative formulation was considered as a performance scale in order to define the best compromise between several requirements. In this paper, a probabilistic design strategy is applied on two kind of stems (solid and IAM stems) to determine the mechanical effects, the response sensitivities with respect to input parameters, and the correlation of the used applied loads with different output parameters. A numerical application on a $2 \mathrm{D}$ problem is carried out to show the advantages of the IAM stem relative to the solid one. The skewness which is considered as an asymmetry measurement of the probability distribution of the output parameters, is evaluated. Next, the sensitivity of the output parameters is performed in order to determine the significant input parameters. Finally, the interdependence (correlation) is carried out for the input and output parameters and also for the input parameters. This concept represents a statistical relationship between two or more parameters such that any modification in the value of one parameter leads to systematic changes in the other (others). The correlation between the input parameters is performed here because of the change of geometry which can affect the loading transfer.

\section{Material and methods}

\subsection{Model description and material properties}

Figs $1 \mathrm{a}$ and $\mathrm{b}$ show 2D sections for the studied solid and IAM stems implanted in bone tissues (cortical and cancellous tissues). Bone materials are generally anisotropic (Mackerle 2006), but in the this study for simplicity, the material properties of bone tissues have been assumed as linearly elastic and isotropic. In Fig. 1, the cortical tissue is assumed to be a homogeneous and isotropic material with Young's modulus $E=17 \mathrm{GPa}$ and Poisson's ratio $v=0.33$. The corresponding number of elements for the cortical region is 605 elements for the solid stem model, while it is 598 elements for the IAM stem model. The cancellous bone tissue is also assumed to be a homogeneous and isotropic material with Young's modulus $E=386 \mathrm{MPa}$ and Poisson's ratio $v=0.33$ (Senapati and Pal 2005). The corresponding number of elements for the cancellous region is 417 elements for the solid and IAM stem models. The elasticity modulus of titanium alloy of stem is considered to be: $E=110 \mathrm{GPa}$ with Poisson's ratio: $v=0.3$ (Shaik et al. 2012). The corresponding number of elements for the metal region is 529 elements for the solid stem model, while it is 784 elements for the IAM 
stem model. The used element is PLANE82 (8-node, nonlinear). The number of the total nodes is 5048 nodes for the solid stem model, while it is 5989 nodes for the IAM stem model.
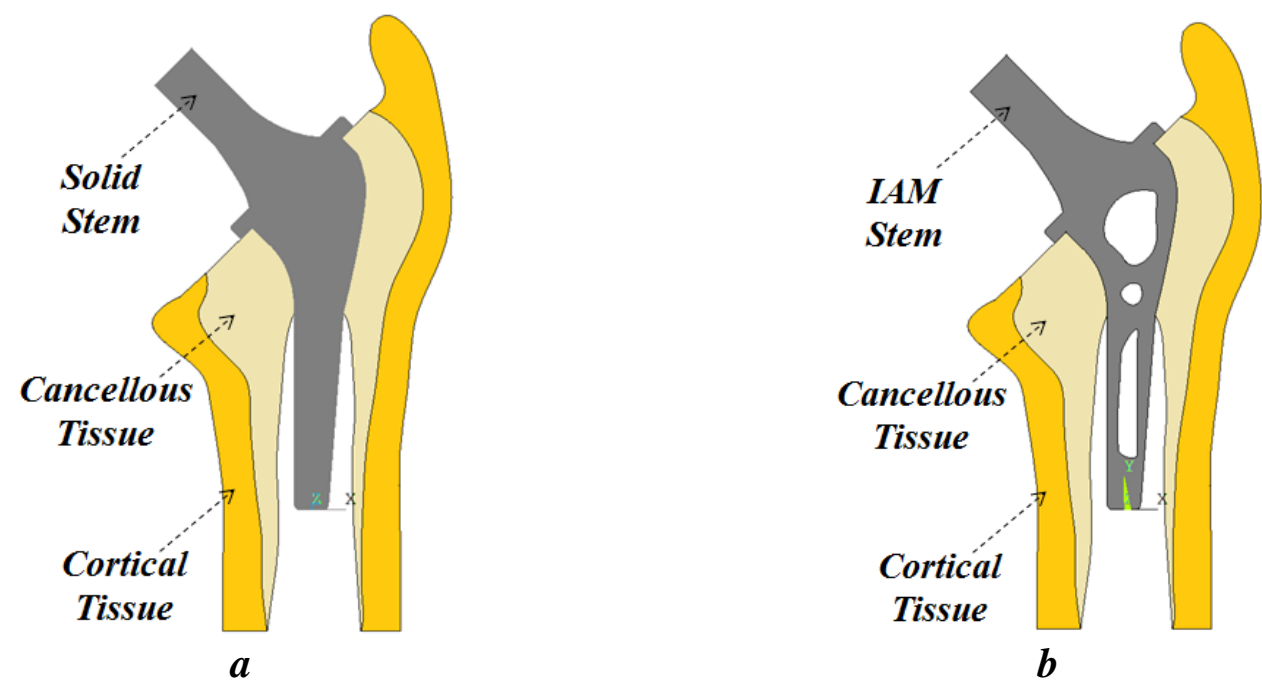

Fig 1. Geometry models for a) solid stem and b) IAM one.

\subsection{Boundary conditions}

According to Beaupré et al. (1990), three loading cases are considered as daily loading conditions: one-legged stance (L1), extreme ranges of motion of abduction (L2), and adduction (L3) as shown in Fig. 2.

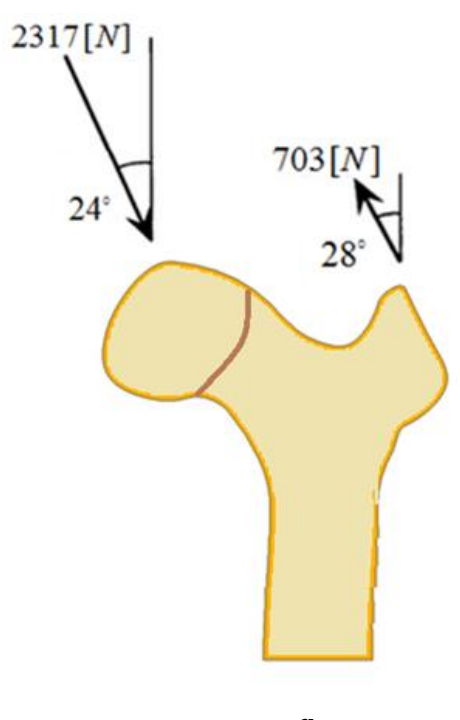

$a$

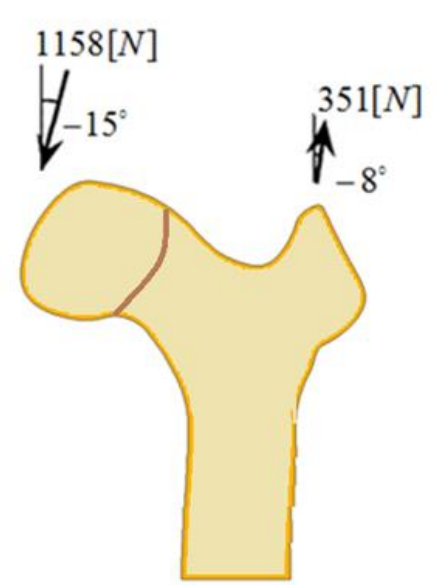

$\boldsymbol{b}$

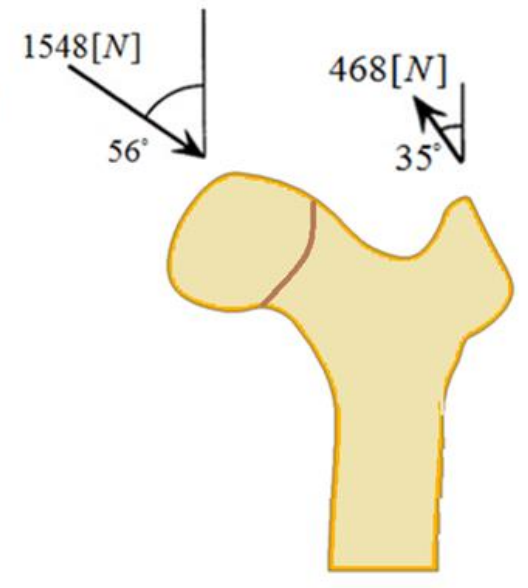

c

Fig. 2. a) One-legged stance case (L1), b) Abduction case (L2), and c) Adduction case (L3).

The component values of applied forces (body and muscles) corresponding to the three loading cases (L1, L2 and L3) which are shown in Fig. 2, are presented in Table 1. 


\begin{tabular}{|c|c|c|c|c|}
\hline \multirow{2}{*}{$\begin{array}{c}\text { Loading } \\
\text { Case }\end{array}$} & \multicolumn{2}{|c|}{ Body Forces } & \multicolumn{2}{c|}{ Muscle Forces } \\
\cline { 2 - 5 } & $F_{x}^{B}[\mathrm{~N}]$ & $F_{y}^{B}[\mathrm{~N}]$ & $F_{x}^{M}[\mathrm{~N}]$ & $F_{y}^{M}[\mathrm{~N}]$ \\
\hline L1 & 942.41 & -2116.68 & -330.04 & 620.71 \\
\hline L2 & -299.71 & -1118.54 & 48.85 & 347.58 \\
\hline L3 & 1283.35 & -865.63 & -268.43 & 383.36 \\
\hline
\end{tabular}

Table 1. Force components (Beaupré et al. 1990).

According to the simulation results in Kharmanda (2016), the third loading case (L3) is the most dangerous case where maximum von-Mises stress values in the bone tissues are the highest values. So, in this work, a probabilistic analysis is carried out considering only this loading case (L3).

\subsection{Probabilistic design strategy}

\subsubsection{Failure probability}

It is the objective to compute the failure probability corresponding to the occurrence of one or several failure modes. The failure probability is then given by

$$
P_{f}=\operatorname{Pr}[G(\mathbf{x}, \mathbf{y})]=\int_{G(\mathbf{x}, \mathbf{y}) \leq 0} f_{Y}(\mathbf{y}) d y_{1} \ldots d y_{n}
$$

where $G(\mathbf{x}, \mathbf{y})$ is a limit state function that is defined by the good functioning conditions of the structure (Kharmanda and El-Hami 2016). The limit state $G(\mathbf{x}, \mathbf{y})=0$ is a separation curve or surface between the failure region $G(\mathbf{x}, \mathbf{y})<0$ and the safety region $G(\mathbf{x}, \mathbf{y})>0 . f_{Y}(\mathbf{y})$ is the density function of the random variable $\mathbf{y}$. Here, many strategies can be used to evaluate the failure probability. Monte Carlo Simulation (MCS) is the most conservative probability technique (Haldar and Mahadevan 2000). It always gives the correct solution if a sufficient number of trials is evaluated, but the computing time consumption is very high (Kharmanda et al. 2018). In order to reduce this time consumption, and avoid overlapping of samples and/or lack of samples in some regions of the domain, Latin Hypercube Sampling (LHS) can be used (McKay et al. 1979). The evaluation of failure probability necessitates a high computing time consumption. So, this technique can be replaced by a reliability index evaluation strategy (Kharmanda et al. 2010; Kharmanda 2015).

\subsubsection{Reliability index}

In general, the failure probability is the inverse of the reliability. The reliability $R$ is related with the failure probability by

$$
P_{f}=1-R
$$

In order to evaluate the reliability index, several method had been elaborated during the last five decades: FORM (First Order Reliability Methods), SORM (Second Order Reliability Method) and simulation techniques (Kharmanda and Antypas 2015; Kharmanda and El-Hami 2016). In FORM, the probability failure is simply approximated by

$$
P_{f} \approx \Phi(-\beta)
$$

where $\Phi(\bullet)$ is the standard Gaussian cumulated function calculated by 


$$
\Phi(Z)=\frac{1}{\sqrt{2 \pi}} \int_{-\infty}^{Z} e^{-\frac{z^{2}}{2}} d z
$$

Equation (3) can give sufficiently accurate assessment of the failure probability, especially when increasing the reliability index values. In the nuclear and spatial applications, very small values of failure probability are necessary. So, the failure probability should be: $P_{f} \in\left[10^{-6}-10^{-8}\right]$. Using Equations 3 and 4, the reliability index should be: $\beta \in[4.75-5.6]$. However, in the structural engineering applications, the failure probability should be: $P_{f} \in\left[10^{-3}-10^{-5}\right]$. Using Equations 3 and 4 , the reliability index should be: $\beta \in[3-4.25]$ (Jeppsson 2003). In the next section, the reliability index is considered to be: $\beta=3$ and the corresponding failure probability is then: $P_{f}=0.13 \%$. The reliability (level of confidence) is $R=99.87 \%$.

\section{Results}

Using ANSYS software, the probabilistic design allows analyzing a component or a system involving uncertain input parameters. Here, the input parameters may concern geometry, material properties, boundary conditions, etc.... These input parameters are considered as random input variables and are characterized by their distribution type (normal, lognormal, uniform, ... etc.) as well as their distribution parameters (mean values, standard deviation, ... etc.). The important responses are selected as random output parameters. The studied model has four random input parameters. Thus, four sources of uncertainty are considered in the present work: $F_{x}^{B}, F_{y}^{B}, F_{x}^{M}$ and $F_{y}^{M}$. It is considered that the input parameters follow the normal distribution law. A high confidence interval of $99.87 \%$ is considered where the failure probability equals $0.13 \%$ and then the reliability index equal to: $\beta=3$ (see Equations 3 and 4). Here, Monte Carlo Simulations are used with 30000 simulations for the third loading case (L3) on the solid and IAM stems. A sensitivity study is performed to assess the influence of each input parameter on the maximum von-Mises stress values for the cortical and cancellous bone tissues, and for the metal $\left(\sigma_{\max }^{1}, \sigma_{\max }^{2}\right.$ and $\left.\sigma_{\max }^{M}\right)$. The interdependence (correlation) between the input parameters and the output parameters is determined. The change of the geometry can affect the input parameters. So, an interdependence study is carried out to find the correlation of between the input parameters.

\begin{tabular}{|c|c|c|c|c|c|c|}
\hline Parameter & Mean & $\begin{array}{c}\text { Standard } \\
\text { Deviation }\end{array}$ & Skewness & Kurtosis & Minimum & Maximum \\
\hline$F_{x}^{B}(\mathrm{~N})$ & 12.83 & 1.283 & $3.5038 \times 10^{-4}$ & $1.1083 \times 10^{5}$ & 7.555 & 18.30 \\
\hline$F_{y}^{B}(\mathrm{~N})$ & 8.656 & 0.8657 & $2.8556 \times 10^{-6}$ & $1.1094 \times 10^{5}$ & 4.890 & 12.49 \\
\hline$F_{x}^{M}(\mathrm{~N})$ & 2.684 & 0.2684 & $3.3388 \times 10^{-5}$ & $1.1088 \times 10^{5}$ & 1.530 & 3.858 \\
\hline$F_{y}^{M}(\mathrm{~N})$ & 3.834 & 0.3833 & $-1.1742 \times 10^{-4}$ & $1.1072 \times 10^{5}$ & 2.273 & 5.409 \\
\hline$\sigma_{\max }^{1}(\mathrm{MPa})$ & 22.36 & 2.960 & $-5.9250 \times 10^{-3}$ & $1.1068 \times 10^{5}$ & 10.25 & 34.42 \\
\hline$\sigma_{\max }^{2}(\mathrm{MPa})$ & 9.507 & 1.161 & $-6.4267 \times 10^{-3}$ & $1.1064 \times 10^{5}$ & 4.709 & 14.25 \\
\hline$\sigma_{\max }^{M}(\mathrm{MPa})$ & 11.28 & 0.8386 & $1.7482 \times 10^{-2}$ & $1.1172 \times 10^{5}$ & 7.914 & 14.68 \\
\hline
\end{tabular}

Table 2. Statistical results of the random input and output parameters for solid stem. 
In Tables 2 and 3, the statistical results of the input and output parameters are presented for the solid stem and for the IAM stem.

\begin{tabular}{|c|c|c|c|c|c|c|}
\hline Parameter & Mean & $\begin{array}{c}\text { Standard } \\
\text { Deviation }\end{array}$ & Skewness & Kurtosis & Minimum & Maximum \\
\hline$F_{x}^{B}(\mathrm{~N})$ & 12.83 & 1.283 & $-6.6698 \times 10^{-5}$ & $1.1072 \times 10^{5}$ & 7.565 & 17.97 \\
\hline$F_{y}^{B}(\mathrm{~N})$ & 8.656 & 0.8657 & $2.1168 \times 10^{-5}$ & $1.1086 \times 10^{5}$ & 4.929 & 12.37 \\
\hline$F_{x}^{M}(\mathrm{~N})$ & 2.684 & 0.2684 & $5.1045 \times 10^{-4}$ & $1.1090 \times 10^{5}$ & 1.562 & 3.869 \\
\hline$F_{y}^{M}(\mathrm{~N})$ & 3.834 & 0.3834 & $-7.2969 \times 10^{-4}$ & $1.1078 \times 10^{5}$ & 2.143 & 5.381 \\
\hline$\sigma_{\max }^{1}(\mathrm{MPa})$ & 22.42 & 2.963 & $5.8377 \times 10^{-4}$ & $1.1082 \times 10^{5}$ & 10.45 & 34.00 \\
\hline$\sigma_{\max }^{2}(\mathrm{MPa})$ & 9.527 & 1.162 & $7.6720 \times 10^{-4}$ & $1.1074 \times 10^{5}$ & 4.878 & 14.10 \\
\hline$\sigma_{\max }^{M}(\mathrm{MPa})$ & 11.29 & 0.8776 & 0.1650 & $1.2140 \times 10^{5}$ & 7.969 & 15.55 \\
\hline
\end{tabular}

Table 3. Statistical results of the random input and output parameters for the IAM stem.

Fig. 3 shows the skewness values of the output parameters $\left(\sigma_{\max }^{1}, \sigma_{\max }^{2}\right.$ and $\left.\sigma_{\max }^{M}\right)$ for the solid and IAM stems.

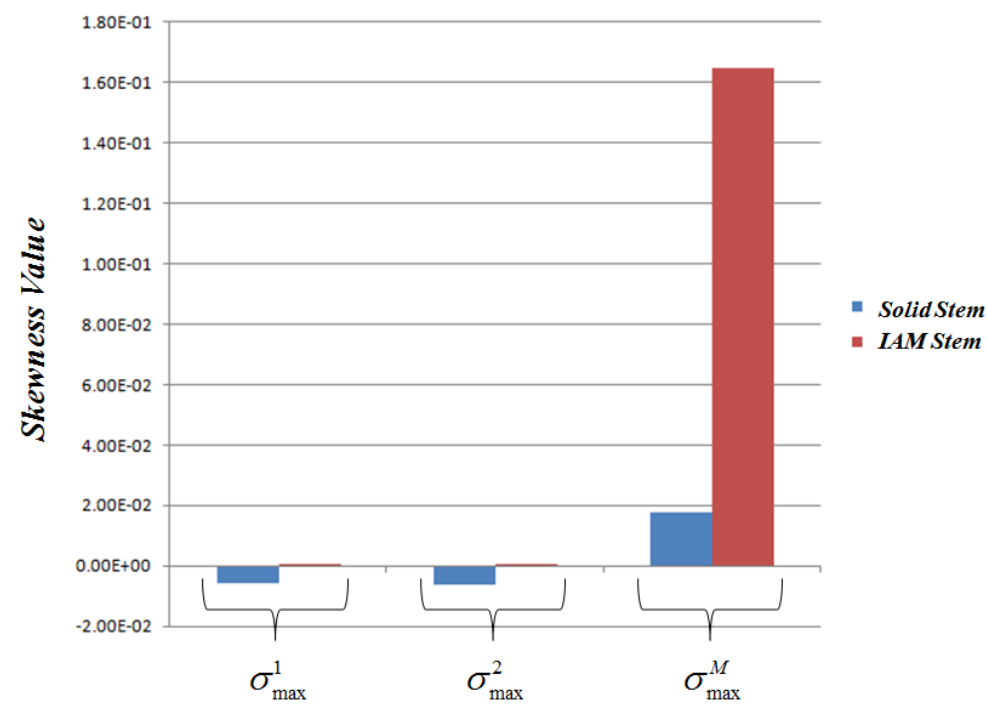

Fig. 3. Skewness values of the different output parameters for the solid and IAM stems.

The sensitivity evaluation of the output parameter with respect to the input random variables are shown in Fig. 4. Fig. $4 \mathrm{a}$ and $\mathrm{b}$ show the sensitivities of the maximum von-Mises stress value $\left(\sigma_{\max }^{1}\right)$ with respect to the significant parameters $\left(F_{x}^{B}, F_{x}^{M}\right.$ and $\left.F_{y}^{M}\right)$ for the solid stem and for the IAM stem, respectively. Fig. $4 \mathrm{c}$ shows the sensitivities of the maximum von-Mises stress value $\left(\sigma_{\max }^{2}\right)$ with respect to the significant parameters $\left(F_{x}^{B}\right.$ and $\left.F_{x}^{M}\right)$ for the solid stem, while Fig. 4d shows the sensitivities of the maximum von-Mises stress value $\left(\sigma_{\max }^{2}\right)$ with respect to the significant parameters $\left(F_{x}^{B}, F_{y}^{B}\right.$ and $\left.F_{x}^{M}\right)$ for the IAM stem. Fig. 4e shows the sensitivities of the maximum von-Mises stress value $\left(\sigma_{\max }^{M}\right)$ with respect to the significant parameters $\left(F_{x}^{B}\right.$ and $\left.F_{y}^{B}\right)$ for the solid stem, while Fig. 4f shows the sensitivities of the maximum von-Mises stress value $\left(\sigma_{\max }^{M}\right)$ with 
respect to the significant parameters $\left(F_{x}^{B}, F_{y}^{B}\right.$ and $\left.F_{x}^{M}\right)$ for the IAM stem. The sensitivities of a certain random output parameter are modeled. The plots of the sensitivities are performed only for the significant random input parameters.
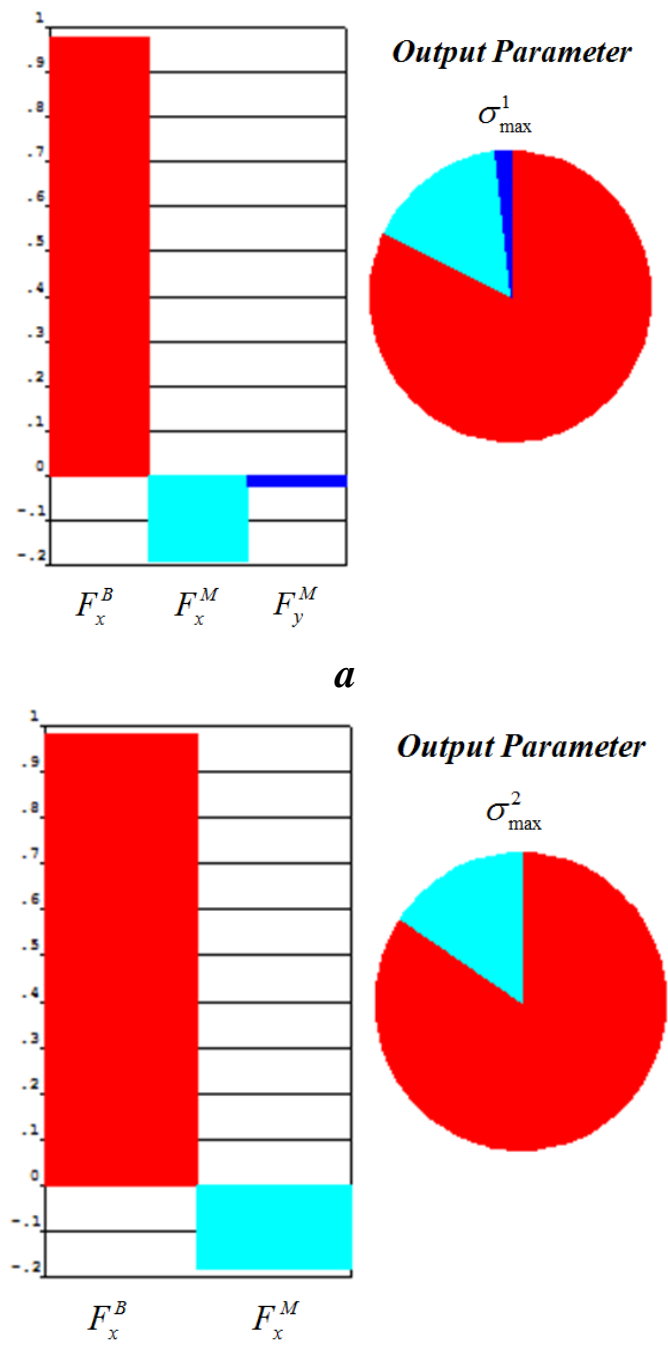

Output Parameter
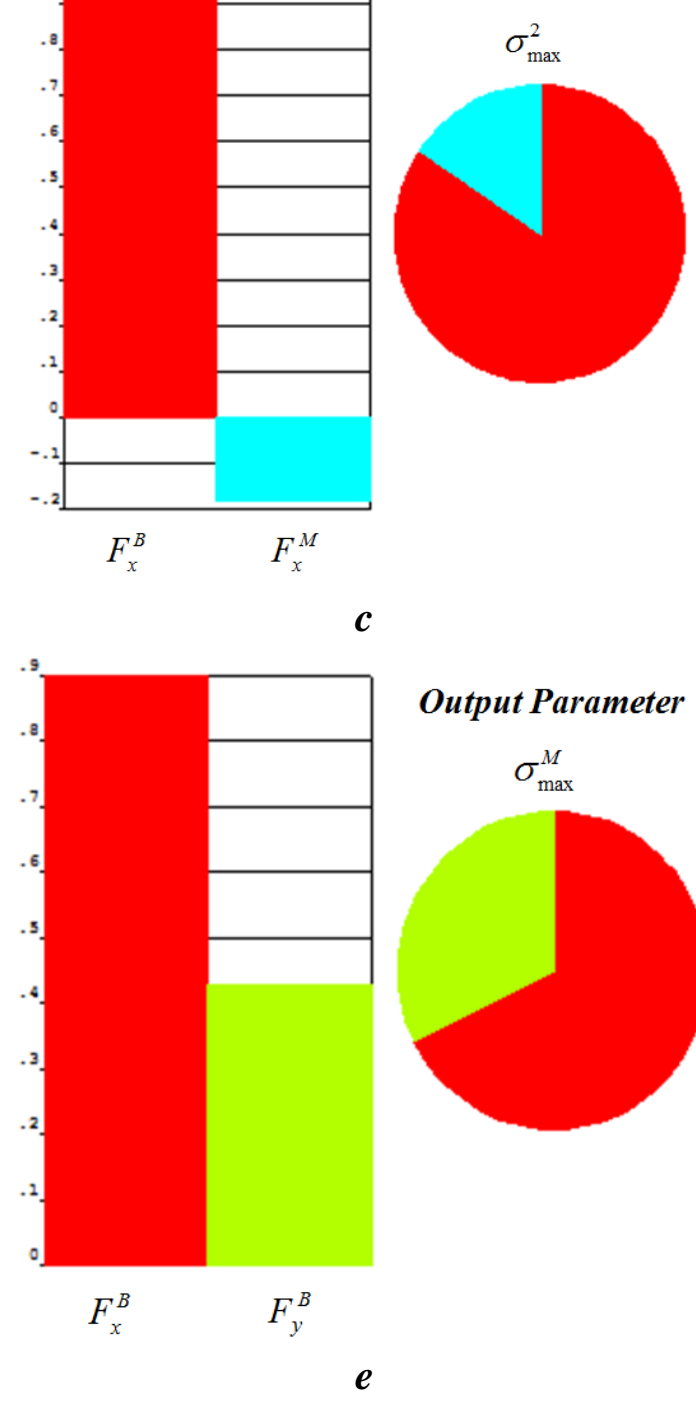

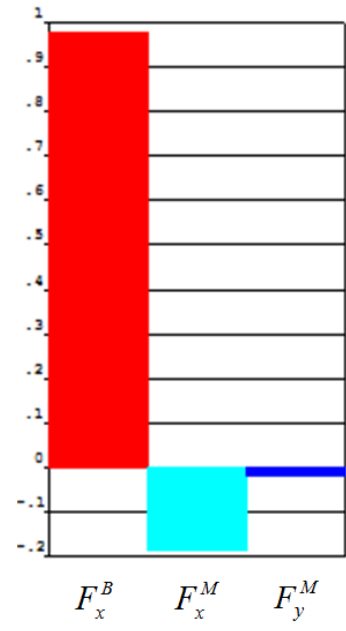

\section{Output Parameter}

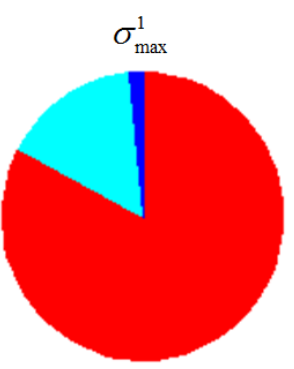

$\boldsymbol{b}$
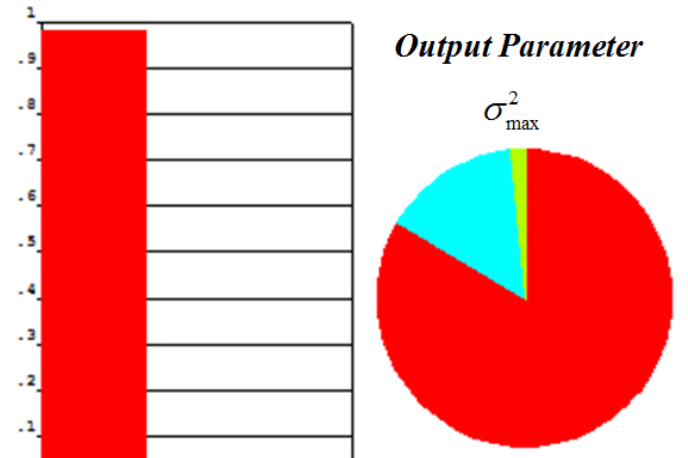

$d$
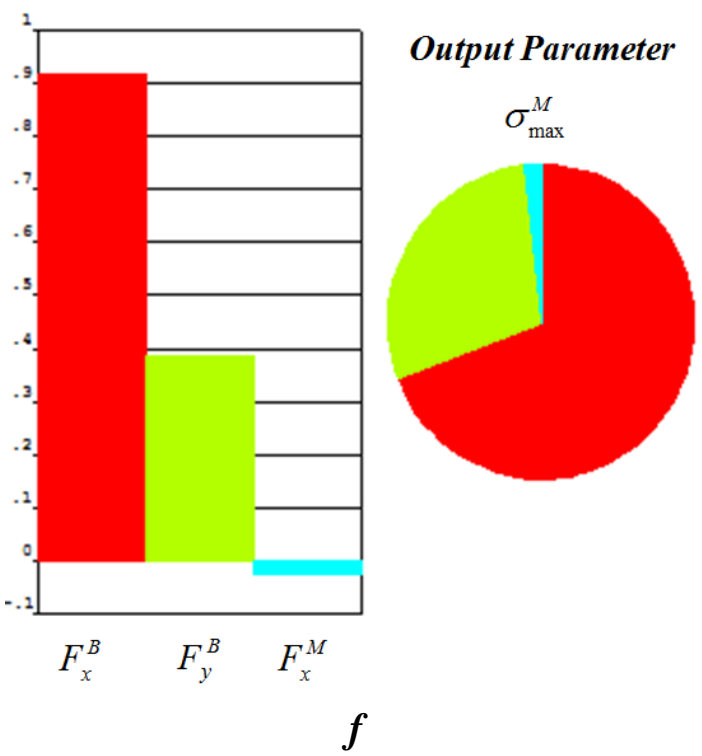

\section{Output Parameter}

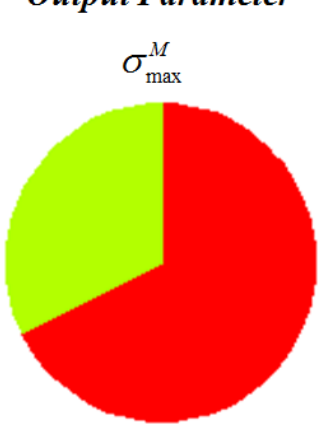

Fig. 4. Sensitivities of the output parameters: $\sigma_{\max }^{1}$ for a) solid stem, and b) IAM stem; $\sigma_{\max }^{2}$ for $c$ ) solid stem, and d) IAM stem; and $\sigma_{\max }^{M}$ for e) solid stem, and f) IAM stem. 
Here, it is shown the statistical interdependence (correlation) between the input and output parameters and also between the inputs parameters. When the correlation coefficient values are close to zero, it means that the two parameters are weakly correlated. On the other hand, when the values are close to 1 or -1 , the two parameters are highly correlated either in positive or negative sense, respectively. Table 4 shows the correlation coefficients between the input and output parameters for the solid and IAM stems.

\begin{tabular}{|c|c|c|c|c|c|c|}
\hline \multirow{2}{*}{ Parameters } & \multicolumn{3}{|c|}{ Solid stem } & \multicolumn{3}{c|}{ IAM stem } \\
\cline { 2 - 7 } & $\sigma_{\max }^{1}(\mathrm{MPa})$ & $\sigma_{\max }^{2}(\mathrm{MPa})$ & $\sigma_{\max }^{M}(\mathrm{MPa})$ & $\sigma_{\max }^{1}(\mathrm{MPa})$ & $\sigma_{\max }^{2}(\mathrm{MPa})$ & $\sigma_{\max }^{M}(\mathrm{MPa})$ \\
\hline$F_{x}^{B}(\mathrm{~N})$ & 0.979 & 0.981 & 0.899 & 0.979 & 0.981 & 0.916 \\
\hline$F_{y}^{B}(\mathrm{~N})$ & -0.011 & 0.014 & 0.428 & -0.003 & 0.022 & 0.384 \\
\hline$F_{x}^{M}(\mathrm{~N})$ & -0.196 & -0.189 & --- & -0.192 & -0.184 & -0.025 \\
\hline$F_{y}^{M}(\mathrm{~N})$ & -0.022 & 0.007 & --- & -0.023 & 0.006 & -0.003 \\
\hline
\end{tabular}

Table 4. Correlation coefficients between the input and output parameters for solid and IAM stems

Table 5 shows the correlation coefficients between the input parameters for the solid and IAM stems.

\begin{tabular}{|c|c|c|c|c|c|c|c|c|}
\hline \multirow{2}{*}{ Parameters } & \multicolumn{5}{|c|}{ Solid stem } & \multicolumn{4}{c|}{ IAM stem } \\
& $F_{x}^{B}(\mathrm{~N})$ & $F_{y}^{B}(\mathrm{~N})$ & $F_{x}^{M}(\mathrm{~N})$ & $F_{y}^{M}(\mathrm{~N})$ & $F_{x}^{B}(\mathrm{~N})$ & $F_{y}^{B}(\mathrm{~N})$ & $F_{x}^{M}(\mathrm{~N})$ & $F_{y}^{M}(\mathrm{~N})$ \\
\hline$F_{x}^{B}(\mathrm{~N})$ & 1.000 & -0.010 & 0.004 & --- & 1.000 & -0.002 & 0.009 & -0.001 \\
\hline$F_{y}^{B}(\mathrm{~N})$ & -0.010 & 1.000 & -0.007 & --- & -0.002 & 1.000 & -0.008 & -0.005 \\
\hline$F_{x}^{M}(\mathrm{~N})$ & 0.004 & -0.007 & 1.000 & --- & 0.009 & -0.008 & 1.000 & 0.003 \\
\hline$F_{y}^{M}(\mathrm{~N})$ & --- & --- & --- & 1.000 & -0.001 & -0.005 & 0.003 & 1.000 \\
\hline
\end{tabular}

Table 5. Correlation coefficients between the input parameters for solid and IAM stems

\section{Discussion}

In this work, a probabilistic analysis is carried out to study in details the role of the different input and output parameters when designing the solid and IAM stems. MCS technique is used as a robust tool with a big number of simulations to provide with accurate results. Three aspects are presented for the solid and IAM stem models: the skewness of the output parameter distributions $\left(\sigma_{\max }^{1}, \sigma_{\max }^{2}\right.$ and $\sigma_{\max }^{M}$ ), the sensitivity of the output parameters with respect to the significant input parameters, and the interdependence (correlation) between the input and output parameters and also between the input parameters.

The skewness values of the output parameters for the IAM stem are different than those for the solid stem. The skewness values of all output parameters of the IAM stem are positive. Positively skewed distribution means that the majority of the maximum von-Mises stress values fall toward the lower side of the scale (their minimum values). However, for the solid stem, the maximum vonMises stress values for the cortical and cancellous tissues $\left(\sigma_{\max }^{1}\right.$ and $\left.\sigma_{\max }^{2}\right)$ have negatively skewed 
distributions, which means that their scores fall toward the higher side of the scale (their maximum values). In addition, when comparing the skewness values of the maximum von-Mises stress values for the metal which have positively skewed distributions for the solid and IAM stem models as shown in Fig. 3, it is noted that the skewness value for the IAM stem model is much higher than that for the solid one.

The sensitivity analysis for the output parameters with respect to the input random parameters is next carried out in order to determine the significant input parameters. Three input parameters $\left(F_{x}^{B}\right.$, $F_{x}^{M}$ and $F_{y}^{M}$ ) has almost same roles on the maximum von-Mises stress values for the cortical tissue $\left(\sigma_{\max }^{1}\right.$ ) for the solid and IAM stems. However, there is a difference when comparing the maximum von-Mises stress values for the cancellous tissue and the metal $\left(\sigma_{\max }^{2}\right.$ and $\sigma_{\max }^{M}$ ). For the solid stem model, only two significant parameters are found: $F_{x}^{B}$ and $F_{x}^{M}$ have influence on $\sigma_{\max }^{2}$ and $F_{x}^{B}$, and $F_{y}^{B}$ have influence on $\sigma_{\max }^{M}$ as shown in Figs $4 \mathrm{c}$ and e. For the IAM stem model, three significant parameters are found: $F_{x}^{B}, F_{y}^{B}$ and $F_{x}^{M}$ have different influence on $\sigma_{\max }^{2}$ and $\sigma_{\max }^{M}$ as shown in Figs $4 \mathrm{~d}$ and $\mathrm{f}$.

According to the correlation (interdependence) study, the correlation coefficient values between the input and the output parameters for the IAM stem are higher than those for the solid stem where the correlation coefficient values between the input parameters $\left(F_{x}^{M}\right.$ and $F_{y}^{M}$ ) and the output

parameter $\sigma_{\max }^{M}$ are close to zero as presented in Table 4. Since the geometry describing the IAM stem is different than that describing the solid one, the loading transfer can be changed. An interdependence (correlation) between the input parameters is then necessary. Table 5 shows that the correlation coefficient values between the input output parameters for the IAM stem are much higher than those for the solid stem where the correlation coefficient values between $F_{y}^{M}$ and the other input parameters $\left(F_{x}^{B}, F_{y}^{B}\right.$ and $\left.F_{x}^{M}\right)$ are close to zero. According to the presented probabilistic design strategy, the IAM stem has several advantages relative to the solid one.

\section{Conclusion}

A probabilistic analysis is performed in order to find the different probabilistic bounds with a high reliability (confidence) level. The results show that the IAM stem is much more advantageous than the solid stem. The present study is limited to a $2 \mathrm{D}$ modeling in order to reduce the computing time since 30000 simulations are performed. In future work, it is recommended to deal with 3D models and to integrate anisotropy behavior for bone tissues instead of bone isotropy. In addition, the variations of the forces are considered to be normally distributed. To improve the study accuracy, statistical data should be introduced. A six sigma methodology can be performed with the aim to determine the extent to which uncertainties in the model affect the results.

\section{References}

Beaupré, G.S.; Orr, T.E.; Carter, D.R. (1990): An approach for time-dependent bone modelingand remodelingapplication: a preliminary remodeling simulation. Journal of Orthopaedic Research 8 (5), 662-670.

Gonzalez, C.D. (2009): Probabilistic Finite Element Analysis of Un-cemented Total Hip Replacement, PhD thesis, School of Engineering Sciences, Bioengineering Sciences Research Group, University of Southampton, March 2009.

Haldar, A.; Mahadevan, S. (2000): Probability, reliability and statistical methods in engineering design. New York, USA: John Willey \& Sons; 2000. 
Huang, T.W.; Huang, K.C.; Lin, S.-J.; Chuang, P.Y.; Shih, H.N.; Lee, M.S. (2016): Effects of teriparatide on cementless bipolar hemiarthroplasty in patients with osteoporotic femoral neck fractures, BMC Musculoskeletal Disorders, 17 (1), pp.300-308, 2016.

Huang, T.W.; Chang, C.H.; Chang, F.C.; Chen, C.C.; Huang, K.C.; Lee, M.S.; Shih, H.N. (2019): Results of Conversion from Failed Austin-Moore Hemiarthroplasty to Cementless Total Hip Arthroplasty in Octogenarian Patients with Advanced Acetabular Erosion: A Minimum of 5 Years of Follow-Up, BioMed Research International Volume 2019, Article ID 7814602, 6 pages https://doi.org/10.1155/2019/7814602.

Jeppsson (2003): Reliability-based assessment procedures for existing concrete structures, PhD dissertation, Division of Structural Engineering, Lund University.

Kanto, K.; Sihvonen, R.; Eskelinen, A.; Laitinen, M. (2014): Uni- and bipolar hemiarthroplasty with a modern cemented femoral component provides elderly patients with displaced femoral neck fractures with equal functional outcome and survivorship at medium-term follow-up, Archives of Orthopaedic and TraumaSurgery,134 (9), pp.1251-1259, 2014.

Kharmanda, G.; El-Hami, A.; Souza de Cursi (2010): Reliability-Based Design Optimization, In edited book Multidisciplinary Design Optimization in Computational Mechanics, Edited by Piotr Breitkopt and Rajan Filomeno Coelho, Chapter 11, : Wiley \& Sons, April 2010, ISBN: 9781848211384 , Hardback 576 pp.

Kharmanda, G.; Albashi, L. (2011): Numerical Study for Increasing Efficiency of Artificially Un-cemented Hip Joint, Research Journal of Aleppo University, Engineering Science Series, vol. 101, 2011.

Kharmanda, G.; Mulki, S.; Sabsabi, Y. (2012): Compter-Aided Design of Internal Replacement Models In Orthopedics Surgery, Research Journal of Aleppo University, series of Engineering Sciences, vol. 105, 2012.

Kharmanda, G.; Ibrahim M.H. (2013): Topology-Based Optimum Design Method for Artificially Replaced Cementless Hip Joints, In the: 10th World Congress on Structural and Multidisciplinary Optimization, WCSMO10, Orlando, Florida, USA, May 19-24, 2013.

Kharmanda, G.; Wallin, M.; Ristinmaa, M. (2014): Integration of multi-objective structural optimization into ANSYS software with application to artificially hip replacement joints, ANSYS 2014 Convergence Conferences, Gothenburg, Sweden, May 20-21, 2014.

Kharmanda, G. (2015): Reliability analysis for cementless hip prosthesis using a new optimized formulation of yield stress against elasticity modulus relationship, Materials and Design (January 2015), 65: 496-504.

Kharmanda, G.; Antypas, I. (2015): Integration of Reliability Concept into Soil Tillage Machine Design, Journal of Don State Technical University 'Vestnik of DSTU', vol. 15 (2), pp 22-31, 2015. ISSN 1992-5980.

Kharmanda, G. (2016): Integration of multi-objective structural optimization into cementless hip prosthesis design: Improved Austin-Moore model, Computer Methods in Biomechanics and Biomedical Engineering, Vol 19 (14), 1557-1566, 2016.

Kharmanda, G.; El-Hami, A. (2016): Reliability Concept, Chapter Nr 2 in book: Reliability in Biomechanics, DOI: 10.1002/9781119370840.ch2, October 2016.

Kharmanda, G.; El-Hami, A. (2017): Biomechanics: Optimization, Uncertainties and Reliability, ISTE \& Wiley, ISBN: 9781786300256, pp 254, January 2017.

Kharmanda, G.; El-Hami, A.; Ibrahim, M.H. (2017): Integration of reliability and optimization concepts into orthopedic prosthesis design: Application on hip prosthesis design, Journal of Uncertainties and Reliability of Multiphysical Systems,17-1, Jan 2017.

Kharmanda G.; Antypas, I. (2018): Reliability-based design algorithm for artificially replaced hip prosthesis considering material property uncertainty, Journal of Advances in Engineering Research, AER, 2018 Advances in Engineering Research (AER), vol 157, pp 44-50.

Kharmanda, G.; Shokry, A.; Antypas, I.; El-Hami, A. (2018): Probabilistic analysis for osseointegration process of hollow stem used in un-cemented hip prosthesis, Journal of Uncertainties and Reliability of Multiphysical Systems, vol 18-2 (2), September 2018.

Kharmanda G.; Antypas, I.; Dyachenko, A. (2019): Effective multiplicative formulation for shape optimization: Optimized Austin-Moore stem for hip prosthesis, International Journal of Mechanical Engineering and Technology, vol 10 (9), pp 1-11, September 2019. 
McKay, M.D.; Beckman, R.J.; Conover, W.J. (1979): A comparison of three methods for selecting values of input variables in the analysis of output from a computer code. Technometrics 1979; 42:55-61.

Mackerle, J. (2006): Finite element modeling and simulations in orthopedics: a bibliography 1998-2005, J. Computer Methods in Biomechanics and Biomedical Engineering, Vol. 9, No. 3, June 2006, 149-199.

Sadoghi, P.; Thaler, M.; Janda, W.; Hubl, M.; Leithner, A.; Labek, G. (2013): Comparative pooled survival and revision rate of austin-moore hip arthroplasty in published literature and arthroplasty register data, The Journal of Arthroplasty, 28 (8), pp.1349-1353, 2013.

Senapati S.K., Pal, S. (2005): UHMWPE-alumina Ceramic Composite: a Proposed Metal Substitute for Artificially Replaced Hip Joint . IE (I) Journal.MC. Vol 85, January 2005.

Shaik, S.A.; Bose, K.; Cherukuri, H.P. (2012): A study of durability of hip implants Materials and Design 42 (2012) 230-237. 